\title{
Supragingival treatment as an aid to reduce subgingival needs: a 450-day investigation
}

\section{Sabrina Carvalho GOMES(a) Rachel ROMAGNA ${ }^{(b)}$ \\ Vanessa ROSSI(b) \\ Paula Chiattone CORVELLO(b) \\ Patrícia Daniela Melchiors ANGST ${ }^{(a)}$}

(a) Department of Periodontology, Dental School, Universidade Federal do Rio Grande do Sul - UFRGS, Porto Alegre, RS, Brazil.

(b) Dental School, Universidade Luterana do Brasil - ULBRA, Canoas, RS, Brazil.

Declaration of Interests: The authors certify that they have no commercial or associative interest that represents a conflict of interest in connection with the manuscript.

Corresponding Author:

Sabrina Carvalho Gomes

E-mail: sabrinagomes.perio@gmail.com

http://dx.doi.org/10.1590/S1806-83242014.50000004 Epub Jan 24, 2014

Submitted: Dec 03, 2012

Accepted for publication: Oct 25, 2013

Last revision: Nov 25, 2013

\begin{abstract}
This study investigated the clinical effects of using a supragingival biofilm control regimen (SUPRA) as a step prior to scaling and root planing (SRP). A split-mouth clinical trial was performed in which 25 subjects with periodontitis ( $47.2 \pm 6.5$ years) underwent treatment (days 0-60) and monitoring (days 90-450) phases. At Day 0 (baseline) treatments were randomly assigned per quadrant: SUPRA, SRP and S30SRP (SUPRA 30 days before SRP). The full-mouth visible plaque index (VPI), gingival bleeding index (GBI), periodontal probing depth (PPD), bleeding on probing (BOP), and clinical attachment loss (CAL) were examined on days $0,30,60,90,120,270$, and 450. Baseline data were similar among all groups. From days 0 to 60 , the groups showed similar significant decreases in VPI and GBI. Reductions in PPD for the $\operatorname{SRP}(3.39 \pm 0.17$ to $2.42 \pm 0.16 \mathrm{~mm})$ and S30SRP $(3.31 \pm 0.11$ to $2.40 \pm 0.07$ $\mathrm{mm})$ groups were greater $(p<0.05)$ than those for the SUPRA group. This pattern was also observed for BOP. Attachment gain was similar and greater for the SRP $(3.34 \pm 0.28$ to $2.58 \pm 0.26 \mathrm{~mm})$ and S30SRP $(3.25 \pm 0.21$ to $2.54 \pm 0.19 \mathrm{~mm})$ groups compared to the SUPRA group. Results were maintained from day 90 forward. Overall, the S30SRP treatment reduced the subgingival treatment needs in $48.16 \%$. Performance of a SUPRA step before SRP decreased subgingival treatment needs and maintained the periodontal stability over time.
\end{abstract}

Keywords: Clinical Trial; Dental Scaling; Root Planing.

\section{Introduction}

Subgingival biofilm control is a sine qua non condition for successful periodontal treatment. ${ }^{1,2}$ However, supragingival biofilm control has been strongly associated with the long-term maintenance of subgingival treatment outcomes. ${ }^{3,4}$ Some authors have noted the importance of the supragingival condition in modulating the subgingival area. ${ }^{5,6}$ However, the exact mechanisms underlying this relationship are not completely understood.

Therapies focused solely on supragingival control ${ }^{78}$ have been demonstrated to significantly reduce subgingival inflammatory markers, such as bleeding on probing (BOP) and periodontal probing depth (PPD). Use of a supragingival biofilm control regimen (SUPRA) reduced the PPD by an average of $2.4 \mathrm{~mm}$ in sites with $6.6 \mathrm{~mm}$ of PPD initially. ${ }^{7}$ This reduction is somewhat comparable or even greater than those achieved by sub- 
gingival instrumentation. For example, Darby et al. ${ }^{9}$ reduced PPD by $1.5 \pm 1.4 \mathrm{~mm}$ in sites with $5.9 \pm 1.3$ $\mathrm{mm}$ of PPD initially, after scaling and root planing (SRP). Similarly, Silva et al. ${ }^{10}$ report a reduction of 1.36 $\mathrm{mm}$ in deep pockets treated with a combination of SRP plus metronidazole and of $1.77 \mathrm{~mm}$ with SRP plus amoxicillin. In addition to improving inflammatory markers, SUPRA ${ }^{7}$ produced significant gains in clinical attachment, which is an important tool for monitoring long-term outcomes. ${ }^{11}$

Despite the importance of these observations, the complementary effects of SUPRA performed before and separately from the SRP intervention remain unexplored. It was hypothesized that SUPRA may be helpful to reduce the subgingival intervention needs. This study evaluated the effect of a supragingival control regimen as a step performed as a prior intervention to SRP.

\section{Methodology}

\section{Study design and sample selection}

This randomized, blinded, split-mouth clinical trial included 25 patients selected consecutively at Universidade Luterana do Brasil - ULBRA (May 2008 to October 2009). The sample size was calculated considering a mean difference of $1.0 \pm 1.0 \mathrm{~mm}$ in PPD reduction, 7 power of $80 \%$, and a of $5 \%$. The inclusion criteria were as follows:

- absence of systemic conditions that could interfere with periodontal indicators (e.g., diabetes, cardiac conditions requiring antibiotic prophylaxis, etc.);

- no chemical control of supragingival biofilm;

- no antibiotic or anti-inflammatory therapy in the 3 months preceding inclusion in the study;

- no pregnancy;

- $\geq 4$ teeth in each quadrant, not including 3rd molars, teeth with furcation involvement, or endoperiodontal lesions;

- $\geq 2$ sites (not in the same tooth and from different quadrants) with a diagnosis of biofilminduced gingivitis and chronic periodontitis, with $\leq 2 \mathrm{~mm}$ difference in PPD and CAL between sites; and

- absence of conditions that could interfere with long-term treatment response.

\section{Ethical considerations}

This study was approved by the Ethics Committee of Universidade Luterana do Brasil (ULBRA; 2007-324H). Participants signed an informed consent form prior to inclusion in the study. Patients were monitored according to progression of clinical attachment loss (CAL). ${ }^{12}$

\section{Experimental interventions}

\section{Treatment allocation}

The following protocols were randomly assigned after the exams on day 0 (baseline). An author who was not involved with the clinical procedures (SCG) drew 2 coins to make the assignments:

- SUPRA (one quadrant): supragingival control as the sole intervention;

- SRP (two quadrants): SUPRA plus subgingival control at day 0 ;

- S30SRP (one quadrant): SUPRA control for 30 days, followed by SRP intervention.

\section{Data collection}

Two examiners who were blinded to group allocation (PC and VR) collected data on days $0,60,90$, 120,270 , and 450 , in the full mouth at 6 sites per tooth. The weighed kappa for interexaminer agreement was 0.75 for PPD and 0.68 for CAL $( \pm 1 \mathrm{~mm})$. The following parameters were assessed: visible plaque (VPI) and gingival bleeding indexes (GBI), ${ }^{13}$ PPD, CAL, and BOP (Williams probe; Neumar, São Paulo, Brazil). The PPD and CAL values were rounded up to the nearest millimeter.

\section{Treatment (days 0-60) and monitoring (days 90-450) phases}

From days 0 to 60 , appointments were conducted by one specialist in periodontics (RR); from days 90 to 450 , they were conducted by another specialist (VR). All appointments were completed within a 45- to 60-minute session. During treatment, SUPRA consisted of calculus removal and oral hygiene instructions. SRP consisted of subgingival hand instrumentation under local anesthesia (Gracey curettes, Hirschfeld files; Neumar, São Paulo, Brazil).

Patients were seen weekly from days 0 to 30 , and then on days 60 and 90 . From day 120 on, they were 
seen at an average interval of $2.2 \pm 1.64$ months. Throughout the study, patients underwent oral hygiene instructions on an individual basis and were given a soft multitufted toothbrush (Colgate Palmolive ${ }^{\circledR}$, São Paulo, Brazil), interdental brush, and/or dental floss, according to their individual needs. No adverse effects were observed during the study.

\section{Statistical analysis}

Three analytic strategies were considered:

1. overall means, considering 6 sites in each tooth;

2. analysis of PPD, BOP, and CAL means, according to PPD categories $(1-3 \mathrm{~mm}, 4-6 \mathrm{~mm}$, and $7+\mathrm{mm})$ at day 0 ; and

3. analysis considering the worst site of each tooth.

Intra- and inter-group comparisons were conducted. An analysis considering the smoking habit was also performed. In case of losses in follow-up, intention-to-treat analysis was used.

Linear models were used to calculate means and standard errors (SEs). Huber-White's sandwich variance estimator was used to adjust SEs for intracluster correlation. Wald's tests were used to estimate $p$-values, which were adjusted for multiple comparisons. All statistical analyses (significance set at $5 \%$ ) were performed by using Stata SE 10.1 software (Stata Corporation, College Station, USA).

\section{Results}

No patients were lost due to CAL progression. Of the 25 patients, three were lost to follow-up, due to changed address, refusal to attend follow-up visits, and pregnancy. Two patients did not attend the 450-day follow-up visit. The carry-forward technique was used in all of these cases, with data collected at the 60- and 270-day visit, respectively, being repeated. The final sample comprised 25 patients (mean age, $47.24 \pm 6.47$ years): 18 females (72\%) and 14 smokers (56\%). In a comparative analysis between smokers and never-smokers, no significant differences were observed for periodontal indicators during the study.

Supragingival assessment revealed significant and similar reductions in the percentage of VPI between days 0 and 60 for all groups. These results were maintained until day 270. At day 450, a difference in VPI was observed between the SRP and S30SRP groups. However, this difference did not have an impact on GBI, which showed no differences between the groups (data not shown).

Table 1 shows the absence of differences in subgingival indicators among all groups at day 0 . All treatments yielded reductions in PPD during the treatment phase (days $0-60 ; p<0.05$ ), with the greatest reductions being observed for the SRP and S30SRP groups. Results were maintained throughout the study. The BOP significantly decreased in all groups, with more pronounced results for the SRP and S30SRP. The CAL data also showed significant overall reductions from 0 to 60 days, which were maintained over the study, with greater reductions observed for the SRP and S30SRP.

When the PPD categories were analyzed (Table 2), the greatest reductions were observed for the SRP and S30SRP groups, with no differences between them. However, the mean PPD values in the SUPRA group were also reduced significantly. This analysis by category also showed improvements in BOP for all groups, with the best results in the SRP and S30SRP for the 4-6 mm and 7+ mm sites. Reductions were maintained throughout the experimental period. At day 450, significant differences were observed between the SUPRA and S30SRP for both the 4-6 $\mathrm{mm}$ and 7+ $\mathrm{mm}$ PPD categories, although the SUPRA and SRP were statistically similar (Table 2). In the 4-6 mm category, gains in clinical attachment were significantly greater in the SRP and S30SRP compared to the SUPRA. The same pattern was observed for the 7+ mm PPD category (Table 2). When only the worst site of each tooth was included in the analysis, no statistical difference between the 3 groups was observed over the study period (Table 3 ).

\section{Discussion}

The present study describes the results from treatment and monitoring phases of 3 periodontal treatment modalities in patients with chronic periodontitis. All treatments achieved significant improvements in periodontal indicators. As expected, groups treated with subgingival intervention (SRP and S30SRP) yielded better results when compared to supragingival control alone, even though the lat- 
Table 1. Mean \pm standard error for periodontal indicators according to the experimental groups during the study.

\begin{tabular}{|c|c|c|c|c|}
\hline Variable & Day & SUPRA & SRP & S30SRP \\
\hline \multirow[t]{7}{*}{ PPD } & 0 & $3.55 \pm 0.19^{\mathrm{Aa}}$ & $3.39 \pm 0.17^{\mathrm{Aa}}$ & $3.31 \pm 0.11^{\mathrm{Aa}}$ \\
\hline & 30 & $3.19 \pm 0.19^{\mathrm{Ab}}$ & $2.71 \pm 0.17^{\mathrm{Bb}}$ & $2.87 \pm 0.12^{\mathrm{Bb}}$ \\
\hline & 60 & $2.86 \pm 0.18^{A c}$ & $2.42 \pm 0.16^{B c}$ & $2.40 \pm 0.07^{B c}$ \\
\hline & 90 & $3.00 \pm 0.19^{A}$ & $2.52 \pm 0.15^{B}$ & $2.36 \pm 0.07^{B}$ \\
\hline & 120 & $2.95 \pm 0.18^{A}$ & $2.45 \pm 0.14^{B}$ & $2.29 \pm 0.07^{B}$ \\
\hline & 270 & $2.78 \pm 0.17^{A}$ & $2.30 \pm 0.11^{\mathrm{B}}$ & $2.17 \pm 0.07^{B}$ \\
\hline & 450 & $2.88 \pm 0.19^{A}$ & $2.36 \pm 0.10^{B}$ & $2.32 \pm 0.08^{B}$ \\
\hline \multirow[t]{7}{*}{$\mathrm{BOP}$} & 0 & $72.38 \pm 3.52^{\mathrm{Aa}}$ & $72.55 \pm 4.58^{\mathrm{Aa}}$ & $72.93 \pm 2.66^{\mathrm{Aa}}$ \\
\hline & 30 & $41.43 \pm 3.70^{\mathrm{Ab}}$ & $25.00 \pm 2.26^{\mathrm{Bb}}$ & $37.61 \pm 3.25^{\mathrm{Ab}}$ \\
\hline & 60 & $31.31 \pm 4.75^{A c}$ & $16.86 \pm 2.90^{B C}$ & $21.15 \pm 2.08^{B C}$ \\
\hline & 90 & $30.25 \pm 4.03^{\mathrm{A}}$ & $30.93 \pm 4.32^{A}$ & $22.00 \pm 2.60^{A}$ \\
\hline & 120 & $27.73 \pm 3.72^{\mathrm{A}}$ & $25.57 \pm 3.46^{A}$ & $21.59 \pm 3.29^{A}$ \\
\hline & 270 & $20.33 \pm 2.50^{A}$ & $17.59 \pm 2.36^{A}$ & $12.31 \pm 1.60^{\mathrm{B}}$ \\
\hline & 450 & $30.91 \pm 4.16^{\mathrm{A}}$ & $20.27 \pm 2.43^{B}$ & $19.88 \pm 2.86^{B}$ \\
\hline \multirow[t]{7}{*}{ CAL } & 0 & $3.50 \pm 0.23^{\mathrm{Aa}}$ & $3.34 \pm 0.28^{\mathrm{Aa}}$ & $3.25 \pm 0.21^{\mathrm{Aa}}$ \\
\hline & 30 & $3.29 \pm 0.23^{\mathrm{Ab}}$ & $2.83 \pm 0.26^{\mathrm{Bb}}$ & $3.03 \pm 0.21^{\mathrm{Ab}}$ \\
\hline & 60 & $3.02 \pm 0.23^{A c}$ & $2.58 \pm 0.26^{B c}$ & $2.54 \pm 0.19^{B C}$ \\
\hline & 90 & $3.06 \pm 0.22^{A}$ & $2.48 \pm 0.22^{B}$ & $2.47 \pm 0.18^{B}$ \\
\hline & 120 & $2.95 \pm 0.21^{A}$ & $2.42 \pm 0.21^{B}$ & $2.41 \pm 0.17^{B}$ \\
\hline & 270 & $2.87 \pm 0.21^{A}$ & $2.38 \pm 0.21^{B}$ & $2.34 \pm 0.17^{B}$ \\
\hline & 450 & $3.02 \pm 0.23^{A}$ & $2.48 \pm 0.22^{B}$ & $2.47 \pm 0.18^{B}$ \\
\hline
\end{tabular}

PPD: periodontal probing depth; BOP: bleeding on probing; CAL: clinical attachment loss; SUPRA: supragingival control; SRP: SUPRA plus scaling and root planing at the same day; S3OSRP: SUPRA and SRP 30 days later; different uppercase letters: significant differences between groups; different lowercase letters: significant differences within group.

ter was also able to promote reductions and to maintain the results over time. Finally, performance of the SUPRA regimen prior to subgingival control (S30SRP) reduced the subgingival needs by about $48 \%$ as measured by the reduction of number of sites with BOP.

The main advantage of the present experimental design is that each patient served as his or her own control, which enabled us to eliminate interindividual variations in periodontal healing. ${ }^{14}$ Hujoel $^{15}$ suggested that, in a split-mouth study, it is important to ensure that sites being submitted to different therapies have the same clinical condition at baseline. In the present study, attention was given to this issue, and subjects were included if they had similar periodontal conditions in quadrants (i.e., $\leq 2 \mathrm{~mm}$ of difference in PPD and CAL values between the sites). The baseline data underscore the similarity between the groups.
A significant reduction in VPI was observed for all groups, which remained low throughout the study. GBI was also reduced in a similar and significant pattern and remained without intergroup differences over the experiment. These findings suggest that the data did not suffer from the carry-across effect; the presence of sites not submitted to SRP did not interfere with the supragingival biofilm formation, which may, in turn, influence the subgingival environment. ${ }^{5-7}$

Full-mouth analysis showed a reduction in all subgingival indicators $(p<0.05)$ for all groups. Although the greatest reductions were detected in groups treated with SRP, supragingival control alone yielded significant reductions, comparable to those observed in other studies that employed subgingival intervention. Santos et al. ${ }^{16}$ observed a mean estimated reduction in PPD of 3.3 to $2.7 \mathrm{~mm}$ after 3 months after SRP plus antibiotics. Our mean PPD 
Table 2. Mean \pm standard error for periodontal indicators according to PPD at baseline.

\begin{tabular}{|c|c|c|c|c|c|c|c|}
\hline \multirow[t]{2}{*}{ Variable } & \multirow[t]{2}{*}{ Day } & \multicolumn{3}{|c|}{$4-6 \mathrm{~mm}$} & \multicolumn{3}{|c|}{$7+\mathrm{mm}$} \\
\hline & & SUPRA & SRP & S3OSRP & SUPRA & SRP & S30SRP \\
\hline \multirow[t]{7}{*}{ PPD } & 0 & $4.67 \pm 0.08^{A a}$ & $4.63 \pm 0.07^{\mathrm{Aa}}$ & $4.67 \pm 0.07^{\mathrm{Aa}}$ & $7.60 \pm 0.15^{\mathrm{Aa}}$ & $7.37 \pm 0.09^{\mathrm{Aa}}$ & $7.28 \pm 0.09^{A C}$ \\
\hline & 30 & $4.01 \pm 0.13^{\mathrm{Ab}}$ & $3.35 \pm 0.17^{\mathrm{Bb}}$ & $3.80 \pm 0.11^{\mathrm{Cb}}$ & $6.87 \pm 0.16^{\mathrm{Ab}}$ & $4.95 \pm 0.38^{\mathrm{Bb}}$ & $6.38 \pm 0.22^{A b}$ \\
\hline & 60 & $3.58 \pm 0.15^{A c}$ & $3.02 \pm 0.17^{\mathrm{Bc}}$ & $2.98 \pm 0.07^{B C}$ & $6.09 \pm 0.24^{A c}$ & $4.24 \pm 0.36^{B c}$ & $4.29 \pm 0.17^{B C}$ \\
\hline & 90 & $3.67 \pm 0.13^{\mathrm{A}}$ & $3.07 \pm 0.17^{B}$ & $2.90 \pm 0.09^{B}$ & $5.80 \pm 0.28^{A}$ & $3.88 \pm 0.29^{B}$ & $3.70 \pm 0.21^{B}$ \\
\hline & 120 & $3.58 \pm 0.14^{\mathrm{A}}$ & $2.93 \pm 0.13^{B}$ & $2.77 \pm 0.10^{B}$ & $5.44 \pm 0.26^{A}$ & $3.69 \pm 0.25^{B}$ & $3.38 \pm 0.24^{B}$ \\
\hline & 270 & $3.40 \pm 0.14^{\mathrm{A}}$ & $2.80 \pm 0.11^{\mathrm{B}}$ & $2.66 \pm 0.10^{\mathrm{B}}$ & $5.11 \pm 0.23^{A}$ & $3.36 \pm 0.21^{\mathrm{B}}$ & $3.19 \pm 0.21^{B}$ \\
\hline & 450 & $3.54 \pm 0.16^{\mathrm{A}}$ & $2.90 \pm 0.10^{\mathrm{B}}$ & $2.84 \pm 0.11^{\mathrm{B}}$ & $5.37 \pm 0.29^{A}$ & $3.42 \pm 0.16^{B}$ & $3.41 \pm 0.19^{B}$ \\
\hline \multirow[t]{7}{*}{$\mathrm{BOP}$} & 0 & $94.05 \pm 1.89^{\mathrm{Aa}}$ & $92.28 \pm 2.04^{\mathrm{Aa}}$ & $90.75 \pm 2.49^{\mathrm{Aa}}$ & $97.86 \pm 1.52^{\mathrm{Aa}}$ & $96.77 \pm 3.19^{\mathrm{Aa}}$ & $97.06 \pm 2.72^{A c}$ \\
\hline & 30 & $59.26 \pm 4.49^{\mathrm{Ab}}$ & $36.58 \pm 3.37^{\mathrm{Bb}}$ & $56.98 \pm 4.28^{\mathrm{Ab}}$ & $85.95 \pm 5.39^{\mathrm{Aab}}$ & $34.40 \pm 4.00^{\mathrm{Bb}}$ & $91.18 \pm 4.78^{A c}$ \\
\hline & 60 & $45.63 \pm 5.47^{\mathrm{Ac}}$ & $23.45 \pm 3.34^{\mathrm{Bb}}$ & $26.89 \pm 2.98^{B c}$ & $83.57 \pm 5.46^{A b}$ & $31.45 \pm 9.45^{\mathrm{Bb}}$ & $42.40 \pm 8.68^{8 b}$ \\
\hline & 90 & $44.23 \pm 5.19^{A}$ & $38.26 \pm 4.30^{A}$ & $26.86 \pm 3.93^{B}$ & $56.86 \pm 9.06^{A}$ & $35.48 \pm 7.96^{A}$ & $36.29 \pm 9.07^{A}$ \\
\hline & 120 & $34.43 \pm 5.73^{\mathrm{A}}$ & $34.30 \pm 4.14^{\mathrm{A}}$ & $32.31 \pm 4.35^{A}$ & $56.86 \pm 8.13^{A}$ & $43.81 \pm 10.19^{\mathrm{AB}}$ & $29.30 \pm 7.08^{B}$ \\
\hline & 270 & $26.45 \pm 4.56^{A}$ & $21.72 \pm 5.01^{\mathrm{A}}$ & $15.24 \pm 2.69^{A}$ & $46.46 \pm 5.69^{A}$ & $23.33 \pm 8.23^{в}$ & $13.22 \pm 2.09 c$ \\
\hline & 450 & $40.52 \pm 5.60^{A}$ & $29.15 \pm 4.52^{\mathrm{AB}}$ & $24.05 \pm 4.60^{B}$ & $56.56 \pm 9.80^{A}$ & $35.00 \pm 7.82^{\mathrm{AB}}$ & $24.19 \pm 9.39^{B}$ \\
\hline \multirow[t]{7}{*}{ CAL } & 0 & $4.59 \pm 0.22^{\mathrm{Aa}}$ & $4.40 \pm 0.23^{\mathrm{Aa}}$ & $4.54 \pm 0.18^{\mathrm{Aa}}$ & $7.30 \pm 0.38^{\mathrm{Aa}}$ & $7.21 \pm 0.32^{\mathrm{Aa}}$ & $7.05 \pm 0.36^{A c}$ \\
\hline & 30 & $4.32 \pm 0.22^{\mathrm{Ab}}$ & $3.55 \pm 0.27^{\mathrm{Bb}}$ & $4.21 \pm 0.19^{\mathrm{Ab}}$ & $6.91 \pm 0.35^{\mathrm{Ab}}$ & $5.82 \pm 0.39^{8 b}$ & $6.79 \pm 0.30^{A b}$ \\
\hline & 60 & $3.93 \pm 0.24^{A c}$ & $3.30 \pm 0.28^{A B C}$ & $3.46 \pm 0.16^{B c}$ & $6.20 \pm 0.33^{A c}$ & $5.39 \pm 0.35^{B c}$ & $5.45 \pm 0.34^{\mathrm{Bb}}$ \\
\hline & 90 & $3.58 \pm 0.27^{A}$ & $2.89 \pm 0.27^{B}$ & $3.18 \pm 0.17^{\mathrm{B}}$ & $5.70 \pm 0.43^{A}$ & $4.75 \pm 0.55^{B}$ & $4.58 \pm 0.30^{B}$ \\
\hline & 120 & $3.45 \pm 0.27^{\mathrm{A}}$ & $2.80 \pm 0.25^{\mathrm{A}}$ & $3.06 \pm 0.15^{A}$ & $5.25 \pm 0.35^{A}$ & $4.33 \pm 0.57^{A B}$ & $4.61 \pm 0.28^{B}$ \\
\hline & 270 & $3.37 \pm 0.28^{A}$ & $2.73 \pm 0.26^{A}$ & $3.00 \pm 0.15^{\mathrm{A}}$ & $5.04 \pm 0.39^{A}$ & $4.30 \pm 0.63^{\mathrm{AB}}$ & $4.36 \pm 0.22^{B}$ \\
\hline & 450 & $3.56 \pm 0.29^{A}$ & $2.83 \pm 0.27^{B}$ & $3.08 \pm 0.16^{B}$ & $5.35 \pm 0.42^{A}$ & $4.36 \pm 0.62^{\mathrm{AB}}$ & $4.24 \pm 0.26^{B}$ \\
\hline
\end{tabular}

Different uppercase letters: significant differences between groups; different lowercase letters: significant differences within group.

for all groups decreased from 3.4 to $2.6 \mathrm{~mm}$ (mean reduction of $0.84 \mathrm{~mm}$ ). Considering only the SRP and S30SRP groups, an even higher mean reduction (1.22 mm) was obtained.

The PPD category analyses showed a similar reduction between groups for the 1-3 $\mathrm{mm}$ sites (data not shown). The results observed for the $4-6 \mathrm{~mm}$ and $7+\mathrm{mm}$ sites showed the greatest reductions in PPD and CAL means at sites submitted to SRP and S30SRP. Nevertheless, at the 4-6 mm sites, SUPRA determined a PPD reduction of $1.09 \mathrm{~mm}$. This mean reduction is similar to that reported by Ioannou et al., ${ }^{17}$ who observed a $1.28 \mathrm{~mm}$ reduction using ultrasonic devices at the subgingival area. In the $7+\mathrm{mm}$ sites, the reductions in PPD $(3.59 \mathrm{~mm})$ and CAL $(2.87 \mathrm{~mm})$ observed for the SRP and S30SRP groups together are comparable to those reported by Santos et al. ${ }^{16}$ with systemic antibiotics $(3.48 \mathrm{~mm}$ and $2.82 \mathrm{~mm}$, respec- tively). Again, the SUPRA quadrants showed obvious reductions, similar to those reported for subgingival treatment. ${ }^{10,17,18}$

A significant improvement in $\mathrm{BOP}$ was observed in all groups. From days 0 to 60 , all groups showed

Table 3. Periodontal indicators considering the worst site of each tooth (day 450 minus day 90).

\begin{tabular}{|l|c|c|c|}
\hline Variable* & SUPRA & SRP & S30SRP \\
\hline \multirow{3}{*}{ PPD } & $-0.22 \pm 0.16^{\mathrm{A}}$ & $-0.20 \pm 0.14^{\mathrm{A}}$ & $-0.10 \pm 0.13^{\mathrm{A}}$ \\
\cline { 2 - 4 } & $\left(p=0.17^{\mathrm{a}}\right)$ & $\left(p=0.16^{\mathrm{a}}\right)$ & $\left(p=0.76^{\mathrm{a}}\right)$ \\
\hline \multirow{3}{*}{ BOP } & $-2.08 \pm 5.58^{\mathrm{A}}$ & $-11.69 \pm 6.81^{\mathrm{A}}$ & $-2.30 \pm 5.17^{\mathrm{A}}$ \\
\cline { 2 - 4 } & $\left(p=0.71^{\mathrm{b}}\right)$ & $\left(p=0.10^{\mathrm{b}}\right)$ & $\left(p=0.66^{\mathrm{b}}\right)$ \\
\hline \multirow{2}{*}{ CAL } & $-0.19 \pm 0.13^{\mathrm{A}}$ & $-0.01 \pm 0.19^{\mathrm{A}}$ & $-0.07 \pm 0.08^{\mathrm{A}}$ \\
\cline { 2 - 4 } & $\left(p=0.15^{\mathrm{c}}\right)$ & $\left(p=0.96^{\mathrm{c}}\right)$ & $\left(p=0.40^{\mathrm{c}}\right)$ \\
\hline
\end{tabular}

${ }^{*}$ mean \pm standard error; negative values mean improvement; different uppercase letters: significant differences between groups; different lowercase letters: significant differences within group. 
a reduction in the percentage of sites with $\mathrm{BOP}$ $(p<0.05)$, although the best results were obtained in the SRP group (reductions from $56.8 \%$ to $76.8 \%$ ). The latter results, which were maintained from day 90 forward, are similar to those reported in a study based on SRP plus antibiotics (reductions from $50 \%$ to $75 \%) .{ }^{18}$ Comparing the percentage of BOP at the 4-6 $\mathrm{mm}$ sites, the present findings showed a reduction from $51.48 \%$ to $74.58 \%$. This pattern was also observed in the 7+ mm sites, where SRP and S30SRP together had a mean reduction of $51.95 \%$, similar to data associated with subgingival control plus antibiotic therapies. ${ }^{18}$

Traditionally, it has been suggested that exclusive supragingival control is not effective for clinical attachment maintenance. ${ }^{19}$ Nevertheless, Gomes et al. ${ }^{7}$ showed that an adequate supragingival regimen permitted clinical attachment gain. In the present study, even sites with deep pockets $(7+\mathrm{mm}$ at baseline) benefited from supragingival control and gained attachment throughout the 450 days.

Overall, considering the presence of BOP as the primary indicator for subgingival intervention, ${ }^{20}$ the treatment applied to the S30SRP group reduced the

\section{References}

1. Jervoe-Storm PM, Semaan E, AlAhdab H, Engel S, Fimmers $\mathrm{R}$, Jepsen S. Clinical outcomes of quadrant root planing versus full-mouth root planing. J Clin Periodontol. 2006 Mar;33(3):209-15.

2. Rhemrev GE, Timmerman MF, Veldkamp I, Van Winkelhoff AJ, Van der Velden U. Immediate effect of instrumentation on the subgingival microflora in deep inflamed pockets under strict plaque control. J Clin Periodontol. 2006 Jan;33(1):42-8.

3. Axelsson P, Nystrom B, Lindhe J. The long-term effect of a plaque control program on tooth mortality, caries and periodontal disease in adults. Results after 30 years of maintenance. J Clin Periodontol. 2004 Sep;31(9):749-57.

4. Heasman PA, McCracken GI, Steen N. Supportive periodontal care: the effect of periodic subgingival debridement compared with supragingival prophylaxis with respect to clinical outcomes. J Clin Periodontol. 2002;29 Suppl 3:163-72; discussion 195-6.

5. Haffajee AD, Teles RP, Socransky SS. The effect of periodontal therapy on the composition of the subgingival microbiota. Periodontol 2000. 2006 Oct;42(1):219-58. number of sites requiring such intervention by $48.16 \%$ compared to the SRP group. At baseline, $72.55 \%$ of the sites in the SRP had BOP and received subgingival SRP (i.e., periodontitis treatment). On the other hand, only $37.61 \%$ of sites from the S30SRP demonstrated BOP after SUPRA and received the SRP intervention. The same comparison in the $4-6 \mathrm{~mm}$ sites showed an important decrease of $38.25 \%$ in subgingival treatment needs (Table 2). As at day 450, the SRP and S30SRP did not differ regarding BOP, but the SUPRA and SRP were similar. Thus, the S30SRP group may have had an even smaller percentage of sites with BOP. This finding underscores that prior well-performed SUPRA control not only reduces subgingival treatment needs, but also maintains this condition over time.

\section{Conclusions}

Adequate supragingival control permits longterm stability in the subgingival environment. If performed as a separate and prior step relative to subgingival intervention, SUPRA may substantially reduce the subgingival treatment needs in patients with gingivitis and periodontitis.

6. Feres M, Gursky LC, Faveri M, Tsuzuki CO, Figueiredo LC. Clinical and microbiological benefits of strict supragingival plaque control as part of the active phase of periodontal therapy. J Clin Periodontol. 2009 Oct;36(10):857-67.

7. Gomes SC, Piccinin FB, Susin C, Oppermann RV, Marcantonio RA. Effect of supragingival plaque control in smokers and never-smokers: 6-month evaluation of patients with periodontitis. J Periodontol. 2007 Aug;78(8):1515-21.

8. Angst PD, Piccinin FB, Oppermann RV, Marcantonio RA, Gomes SC. Response of molars and non-molars to a strict supragingival control in periodontal patients. Braz Oral Res. 2013 Jan-Feb;27(1):55-60.

9. Darby IB, Mooney J, Kinane DF. Changes in subgingival microflora and humoral immune response following periodontal therapy. J Clin Periodontol. 2001 Aug;28(8):796-805.

10. Silva MP, Feres M, Sirotto TA, Soares GM, Mendes JA, Faveri $\mathrm{M}$, et al. Clinical and microbiological benefits of metronidazole alone or with amoxicillin as adjuncts in the treatment of chronic periodontitis: a randomized placebo-controlled clinical trial. J Clin Periodontol. 2011 Sep;38(9):828-37. 
11. Mombelli A. Clinical parameters: biological validity and clinical utility. Periodontol 2000. 2005 Oct;39(1):30-9.

12. Haffajee AD, Socransky SS, Goodson JM. Comparison of different data analyses for detecting changes in attachment level. J Clin Periodontol. 1983 May;10(3):298-310.

13. Ainamo J, Bay I. Problems and proposals for recording gingivitis and plaque. Int Dent J. 1975 Dec;25(4):229-35.

14. Lesaffre E, Garcia Zattera MJ, Redmond C, Huber H, Needleman I. Reported methodological quality of split-mouth studies. J Clin Periodontol. 2007 Sep;34(9):756-61.

15. Hujoel PP. Design and analysis issues in split mouth clinical trials. Community Dent Oral Epidemiol. 1998 Apr;26(2):85-6.

16. Santos VR, Ribeiro FV, Lima JA, Miranda TS, Feres M, Bastos $\mathrm{MF}$, et al. Partial- and full-mouth scaling and root planing in type 2 diabetic subjects: a 12-mo follow-up of clinical parameters and levels of cytokines and osteoclastogenesis-related factors. J Periodontal Res. 2012 Feb;47(1):45-54.
17. Ioannou I, Dimitriadis N, Papadimitriou K, Sakellari D, Vouros I, Konstantinidis A. Hand instrumentation versus ultrassonic debridement in the treatment of chronic periodontitis. A randomized clinical and microbiological trial. J Clin Periodontol. 2009 Feb;36(2):132-41.

18. Feres M, Haffajee AD, Allard K, Som S, Socransky SS. Change in subgingival microbial profiles in adult periodontitis subjects receiving either systemically-administered amoxicillin or metronidazole. J Clin Periodontol. 2001 Jul;28(7):597-609.

19. Kaldahl WB, Kalkwarf KL, Patil KD, Molvar MP, Dyer JK. Long-term evaluation of periodontal therapy: I. Response to 4 therapeutic modalities. J Periodontol. 1996 Feb;67(2):93-102.

20. Brochut PF, Marin I, Baehni P, Mombelli A. Predictive value of clinical and microbiological parameters for the treatment outcome of scaling and root planing. J Clin Periodontol. 2005 Jul;32(7):695-701. 